\title{
Intrahepatic cholestasis of pregnancy
}

\author{
Timely intervention reduces perinatal mortality
}

Intrahepatic cholestasis of pregnancy is one of the few disorders that adversely affect maternal wellbeing and fetal outcome. Early diagnosis and timely intervention can reduce perinatal mortality.

The condition is the most common liver disorder peculiar to pregnancy and is second to viral hepatitis as a cause of jaundice in the third trimester. It is rarely reported except in Chile, Bolivia, Scandinavia, and China. ${ }^{1}$ Its true incidence and range are unknown. Generalised pruritus, mild jaundice, and intrahepatic cholestasis in late pregnancy were reported by Ahlfeld in $1883^{2}$ and later by Eppinger ${ }^{3}$ and Thorling. ${ }^{4}$ Svanborg also described fatigue and mild abdominal pain and suggested that the condition resolved rapidly after birth but tended to recur in successive pregnancies. 5 Pruritus typically develops after 20 weeks of pregnancy; is prominent on the arms, legs, and trunk; and progresses until delivery. Jaundice may develop two to four weeks later without progressing.

The pathogenesis of intrahepatic cholestasis of pregnancy is unclear. Multiple factors probably interact with a genetic predisposition to alter the membrane composition of bile ducts and hepatocytes and increase their sensitivity to sex steroids. ${ }^{1}$ In cholestasis, itching has been linked to increased availability of opiate receptors in the brain to bind their agonist ligands. ${ }^{6} \mathrm{~A}$ family history (autosomal dominant and possibly $\mathrm{X}$ linked) is commonly associated with haplotypes HLA-B8 and HLA-Bw16. Fathers transmit the susceptibility to daughters. Hormonal factors are implicated because intrahepatic cholestasis worsens with multiple pregnancies and can recur with menstruation and oestrogen treatment. Cholestasis and pruritus may resolve after high dose $S$-adenosyl-L-methionine, suggesting a metabolic defect. Seasonal variation in countries with a high prevalence suggests that environmental factors could have a role.

Reports tend to contrast the low maternal mortality with the high perinatal mortality, but maternal morbidity can be considerable. Nocturnal itching causes insomnia and fatigue. Anorexia, malaise, mild epigastric discomfort, steatorrhoea, and dark urine are common. Malabsorption of fat can lead to weight loss and vitamin deficiency, particularly vitamin $\mathrm{K}$ deficiency, which may account for some cases of uterine and intracranial haemorrhage.

Jaundice is an uncommon presenting feature. Serum concentrations of conjugated bilirubin and activities of alkaline phosphatase, aspartate aminotransferase, and alanine aminotransferase are rarely higher than three times the normal values. Other causes of pruritus and jaundice require exclusion, especially gall stones, primary biliary cirrhosis, sclerosing cholangitis, viral hepatitis, autoimmune chronic active hepatitis, and drug hepatotoxicity. Severe pain, hepatomegaly, splenomegaly, or fever suggests other causes. Ultrasonographic imaging helps to exclude gall stones and biliary disease and to locate the gall bladder, which is exceptionally large in intrahepatic cholestasis of pregnancy, before percutaneous biopsy. Histological confirmation of acinar cholestasis and bile plugs is unnecessary except in atypical cases when symptoms start before 20 weeks, jaundice precedes pruritus, and itching persists after delivery.

Women with intrahepatic cholestasis of pregnancy, a history of familial cholestasis, or jaundice exacerbated by oestrogens should be followed up closely throughout pregnancy. Serial measurements of maternal serum concentrations of bile acids, albumin, and alkaline phosphatase are essential. Steatorrhoea can be overlooked unless body weight and faecal fats are measured objectively. Supplementation of fat soluble vitamins, particularly vitamin $K$, may be required.

Maternal outcome is good. Itching and jaundice resolve rapidly after delivery but may recur with subsequent pregnancies, menstruation, and oestrogen treatment. Substances for relieving itching, such as cholestyramine, phenobarbitone, charcoal, ultraviolet light, evening primrose oil, intravenous $S$-adenosyl-L-methionine, and epomediol, have proved disappointing in trials but may benefit individual women. Cholestyramine binds bile acids, anionic drugs, and fat soluble vitamins, and vitamin $K_{1}$ may be needed to reduce the risk of postpartum haemorrhage. The use of phenobarbitone and antihistamines can aggravate the respiratory difficulties of a preterm baby by causing sedation. Ursodeoxycholic acid, a naturally occurring hydrophilic bile salt, has been shown in small studies to reduce biochemical abnormalities and itching without adversely affecting the newborn baby. ${ }^{78}$ It can relieve itching in chronic cholestatic syndromes, but in Britain its use is contraindicated in pregnancy. Bile acids cross the placenta, and concern remains over their potential teratogenicity in some animal studies, though in these studies they were given in early pregnancy.

The management of intrahepatic cholestasis in pregnancy is overshadowed by the increased risks of fetal distress, spontaneous preterm delivery, and death. ${ }^{19}$ The causes of these are unknown. Risks are unrelated to the 
severity of symptoms and increase near term. Close monitoring of fetal wellbeing is essential, although no single test reliably predicts the risk of intrauterine death. Raised serum concentrations of maternal bile acids correlate with the severity of pruritus and risk of fetal distress. ${ }^{10}$ Some obstetricians recommend regular non-stress and contraction stress tests. Delivery should ideally take place around 38 weeks after serial estimations of fetal lung maturity and maternal serum concentrations of total bile acids. Delivery around 36 weeks should be considered for severe intrahepatic cholestasis of pregnancy with jaundice and progressive increases in serum bile acid concentrations if fetal distress is suspected and lung maturity has been confirmed. ${ }^{19}$ The intrapartum fetal heart rate seems an unreliable indicator of fetal distress in intrahepatic cholestasis of pregnancy. ${ }^{9}$ Giving vitamin $K_{1}$ to the baby immediately after birth may help prevent intracranial bleeding.

Recent reports of optimistic fetal outcomes are mostly from experienced referral centres in countries with a high prevalence of the condition. ${ }^{19}$ Maternal and fetal wellbeing are likely to improve with a better understanding of the pathogenetic mechanisms associated with bile acid metabolism, pruritus, and cholestasis. Treatment will be hampered by insufficient data on drug toxicity during late pregnancy in humans.

ELIZABETH ANN FAGAN Visiting professor

University Gastroenterologists,

Rush Presbyterian St Luke's Medical Center,

Chicago, IL 60612,

USA

1 Reyes $\mathrm{H}$, Simon FR. Intrahepatic cholestasis of pregnancy: an estrogen-related disease. Sem Liver Dis 1993;13:289-301.

2 Ahlfeld F.Berichte und Arbeiten aus der geburtshilfich-gynaekologischen Klinik zu Giessen 18811882. Leipzig: Grunow, 1883:148.

3 Eppinger H. Die Leberkrankheiten; allgemeine und spezielle Pathologie und Therapie der Leber. Vienna: Springer-Verlag, 1937.

4 Thorling L. Jaundice in pregnancy. A clinical study. Acta Med Scand 1955;302 (suppl):1-123. 5 Svanborg A. A study of recurrent jaundice in pregnancy. Acta Obstet Gynecol Scand 1954;22:434-44.

6 Jones EA, Bergasa NV. The pruritus of cholestasis and the opioid system. FAMA 1992;268:3359-62.

7 Palma J, Reyes H, Ribalta J, Iglesias J, Gonzalez MC, Hernandez I, et al. Effects of ursodeoxycholic acid in patients with intrahepatic cholestasis of pregnancy. Hepatology 1992;15: 1043-7.

8 Floreani A, Grella V, Gangemi M. Ursodeoxycholic acid in intrahepatic cholestasis of pregnancy. Br f Obstet Gynaecol 1994;101:64-5.

9 Rioseco AJ, Ivankovic MB, Manzur A, Hamed F, Kato SR, Parer JT, et al. Intrahepatic cholestasis of pregnancy: a retrospective case-control study of perinatal outcome. Am 7 Obstet Gynecol 1994;170:890-5.

10 Laatikainen T, Tulenheiko A. Maternal serum bile acid levels and fetal distress in cholestasis of pregnancy. Int $₹$ Gynaecol Obstet 1984;22:91-4.

\section{Pulmonary emphysema}

\section{We now understand more about the causes, but effective treatment is some way off}

The treatment of advanced pulmonary emphysema is a serious and depressing problem for respiratory physicians. The patients, mainly smokers or former smokers, suffer from progressive disability and have a reduced life expectancy. Such patients are short of breath on mild exertion and have hyperresonant chests and quiet or absent breath sounds. Destruction of the alveolar walls is a key feature of the disease. ${ }^{1}$

Emphysema can be divided into pathological types according to the distribution of the enlarged air spaces within the acinus (the section of the lung that is supplied by a single terminal bronchiole ${ }^{2}$ ). Two types seem important. In the centriacinar form the enlarged air spaces are in the centre of the acinus close to the terminal bronchioles, and these lesions are usually situated in the upper zones of the lungs. In the panacinar form the whole acinus is affected and lesions may be distributed throughout the lung or concentrated in the lower zones.

The pathogenesis of emphysema remained obscure until Swedish workers discovered the association between panacinar emphysema and a hereditary deficiency of $\alpha_{1}$-proteinase inhibitor. ${ }^{3} \alpha_{1}$-Proteinase inhibitor inhibits proteolytic enzymes, the most important being neutrophil elastase, which can degrade most of the structural proteins of the lung as well as elastin. $\alpha_{1}$-Proteinase inhibitor protects the lung against such proteolytic damage. It is made in the liver and is normally present in the serum in high concentration, but in $\alpha_{1}$-proteinase inhibitor deficiency an error in genetic coding prevents its release from the liver in adequate quantities.

Neutrophils and macrophages can release proteolytic enzymes capable of degrading pulmonary tissue. In smokers and in the presence of infection both cell types increase in number and activity. The main macrophage enzymes are the cysteine proteinases, which are inhibited by the cystatins, and the metalloproteinases, which are inhibited by the specific tissue inhibitors of metalloproteinase. The macrophage enzymes are probably responsible for centriacinar emphysema, in which the lesions seem to be caused by an agent arising in the lumen or the walls of the airways. Neutrophil enzymes are likely to cause the panacinar lesions, the distribution of which suggests that they are caused by a bloodborne agent.

An excess of enzyme or lack of inhibitor is thought to allow structural damage to the lung. This theory receives much additional support from experimental data; lesions similar to those seen in humans with emphysema can be produced in animals by instillation of proteolytic enzymes into their airways. ${ }^{4}$

Nearly all patients with emphysema who have a normal $\alpha_{1}$-proteinase inhibitor phenotype are cigarette smokers, and in patients with $\alpha_{1}$-proteinase inhibitor deficiency smoking greatly increases the severity of the condition. Cigarette smoke could cause emphysema by stimulating neutrophils and macrophages to increase production of proteinases; stimulating them to produce oxidants such as hypochlorous acid, which can inactivate the proteinase inhibitors; suppressing lysyl oxidase, an enzyme required for the synthesis of elastin; or depositing cadmium in the lung, which itself can cause emphysema. Susceptibility to tobacco smoke, however, varies widely as only $10-20 \%$ of smokers develop emphysema. ${ }^{5}$

The theory of proteinase-antiproteinase balance has not gone unchallenged, with suggestions that airflow obstruction in adults is mainly determined in infancy and childhood or even in utero. In animal experiments maternal energy deprivation or hypoxia can reduce fetal pulmonary elastin, which in humans might be presumed to cause emphysema in later life. This question has been addressed by studies in which birth weights recorded 60 to 70 years ago were used as a marker for fetal lung growth. ${ }^{6}$ The mean forced expiratory volume in one second was 